\title{
Efficiency of Multi Detector Computed Tomography in Staging of Gastric Carcinoma and Detection of Distant Metastatic Disease
}

\author{
R Vysakh ${ }^{1}$, Varghese Joseph ${ }^{2}$ \\ ${ }^{1}$ Junior Resident, Department of General Surgery Amala Institute of Medical Sciences Amala Nagar, Thrissur, \\ ${ }^{2}$ Assistant Professor, Department of General Surgery, Amala Institute of Medical Sciences Amala Nagar, Thrissur, \\ India
}

Corresponding author: Dr. Varghese Joseph, Assistant Professor of General Surgery Department of General Surgery, Amala Institute of Medical Sciences Amala Nagar, Thrissur-680 555, India

DOI: http://dx.doi.org/10.21276/ijcmsr.2019.4.4.17

How to cite this article: $\mathrm{R}$ Vysakh, Varghese Joseph. Efficiency of multi detector computed tomography in staging of gastric carcinoma and detection of distant metastatic disease. International Journal of Contemporary Medicine Surgery and Radiology. 2019;4(4):D71-D74.

\section{A B S T R A C T}

Introduction: A proper preoperative evaluation is essential to plan the surgery to attain the aim of a RO resection. This study was aimed at comparing the efficiency of preoperative staging of gastric cancer using Contrast Enhanced Computed Tomography (CECT) scan by assessing tumor location, depth of invasion, lymph node involvement and adjacent organ involvement as compared to post-operative histopathological staging.

Material and Methods: All patients with histopathologically confirmed gastric malignancy by endoscopy underwent CECT for preoperative staging. The finding was compared with post-operative histopatholgic findings. The findings were analysed for sensitivity, specificity, positive predictive value and negative predictive value of CECT for T staging, detecting nodal status and adjacent organ involvement.

Results: The mean age of the patients were 58.9 years with $61 \%$ patients being males. All patients included had serosal involvement at the time of presentation and all of them were picked up by CECT. The ability of CECT to detect sub layer involvement could not be assessed. For N staging, CECT was less sensitive while for N1 disease, maximum specificity of $94.44 \%$. For N2 disease, it showed maximum sensitivity of $55.55 \%$. On evaluating the adjacent organ involvement CECT showed least sensitivity for bowel involvement (16.66\%) and maximum sensitivity for pancreatic involvement (71.43\%).

Conclusion: CECT remains poor at identifying lymph node metastases and in determining depth of tumour invasion (T1T3). But it was highly specific which meant that a positive spiral CT result could be relied upon. It was also effective in identifying the invasion of pancreas and in some cases of distant metastatic disease.

Keywords: Contrast Enhanced Computed Tomography, Gastric Cancer, Preoperative Evaluation, Sensitivity, Specificity.

\section{INTRODUCTION}

Gastric cancer describes a broad mix of malignant neoplasms derived from the different histological components that make up the stomach. These include adenocarcinoma, lymphoma, carcinoid tumours and sarcoma. Among these, gastric adenocarcinoma accounts for over $90 \%$ of all cases of gastric cancers globally. According to 2014 cancer statistics, gastric cancer is the second most common cause of gastrointestinal malignancy, in both, newly detected case and cancer related deaths in U.S.1 The worldwide incidence of gastric cancer has declined rapidly over the recent few decades. Despite its declining incidence, it is the third most common cause of cancer-related mortality globally. ${ }^{1}$ The decline may be partially due to the recognition of certain risk factors such as $H$. pylori and other dietary and environmental risks. ${ }^{2}$ The gastric cancer prevalence in India is 5.14 for both sexes (male: 5.14, females: 3.34). The mortality rates according to GLOBOCAN registry is 5.65 for both sexes (male:8 and female: 3.4).3 Weight loss has been considered among the potential prognostic factors for gastric cancer by at least 13 studies so far, and in 10 of these it significantly and independently related to the fatal outcome. ${ }^{4-8}$ Delay in diagnosis was longer for patients without alarm symptoms compared to that of patients with alarm symptoms. ${ }^{4-6}$ Neoadjuvant treatment is recommended for any gastric cancer patient without metastatic disease who is either node positive or staged as T2 or greater in the preoperative setting. It is of great importance for the operating surgeon to know the adjacent organ involvement to obtain an R0 resection. Despite increased referral for consideration of surgical treatment, $15-30 \%$ of patients have unresectable disease and undergoes no procedure other than open biopsy. Traditionally, staging has been performed at the time of surgery, although a sensitive and reliable staging tool which 
could identify patients with unresectable disease would be of great benefit in saving patients from unnecessary laparotomy. Contrast Enhanced Computed Tomography (CECT) scan of abdomen and pelvis is the most commonly used investigation for pre-operative staging. But its value remains controversial. So this study was aimed at comparing the CECT staging with post operative histopathological staging in carcinoma stomach.

\section{MATERIAL AND METHODS}

Descriptive cross sectional study was conducted among patients who attended the General Surgery and Surgical Oncology departments of Amala Institute of Medical Sciences, Thrissur, Kerala from January, 2015 to July, 2016. Histopathology reports and CT scan reports were collected from Pathology and Radiology departments respectively. Both males and females of age between 35 and 75 years who were provisionally diagnosed as gastric carcinoma (using endoscopic biopsy) were included in the study. Patients who had undergone neo-adjuvant treatment or presented with an acute abdomen or recurrences and patients aged less than $35 \mathrm{yrs}$ and more than 75 yrs were excluded from the study. Sample size-61 was calculated based on the previous study where $48 \%$ sensitivity for CT scan in the preoperative staging of gastric cancer was reported and also based on the prevalence of the disease assumed to be $6.3 \% .^{9}$

\section{Procedure of the study}

All patients with inclusion criteria during the study period were taken up for study. An informed consent was obtained from the patient. Pre operative staging was done using CECT of abdomen and pelvis. All necessary pre operative investigations were done in patients selected for surgery. Post operative histopathological specimen which included gastrectomy specimen along with relevant lymph node stations; and biopsy/cytology specimen from suspicious lesion in adjacent organs like peritoneum, omentum, liver, serosal surface of bowel and pelvic deposits were obtained. Histopathological data included size, location, depth of invasion, lymph node status and biopsy proven distant metastasis.

\section{STATISTICAL ANALYSIS}

Statistical analysis was done using SPSS-23 software. Sensitivity, specificity, positive predictive value and negative predictive value of preoperative Multi Detector CECT was estimated individually for location, depth of invasion, lymph node status and adjacent organ spread. Analysis was done using Chi Square test. $\mathrm{P}<0.05$ was considered as significant.

\section{RESULTS}

Distribution of age of the patients is depicted in table 1. Among the total cases, $28(45.6 \%)$ cases were in the age group of 61-70 years. The mean age of the study group was $58.9 \pm 10.3$ years. Males patients were $37(60.7 \%)$ and females were $24(39.3 \%)$ with a ratio of $1.54: 1$. All of the 61 cases had T4 disease. Pre-operative CECT evaluation showed 4.9\% with N1 disease, $50.8 \%$ with N2 disease, $24.6 \%$ with N3 disease and $19.7 \%$ with N4 disease (Table 2).

CECT identified involvement of $\mathrm{N} 1$ lymph nodes in $0 / 7$ patients, giving a sensitivity of 0 . However, CT correctly identified 51/54 patients who did not have nodal involvement (specificity 94.44\%). Operative assessment of N1 nodal status was superior to that of CECT, correctly identifying 1 of the 7 cases (sensitivity $14.29 \%$ ), but with a specificity of $100 \%$ (54/54) identifying all 54 patients without N1 disease status

\begin{tabular}{|l|c|c|}
\hline Age & Frequency & Percent \\
\hline$\leq 40$ & 3 & 4.9 \\
\hline $41-50$ & 10 & 16.4 \\
\hline $51-60$ & 16 & 26.2 \\
\hline $61-70$ & 28 & 45.9 \\
\hline$\geq 71$ & 4 & 6.6 \\
\hline Total & 61 & 100.0 \\
\hline \multicolumn{2}{|c|}{ Table-1: Distribution of age } \\
\hline
\end{tabular}

\begin{tabular}{|l|c|c|c|c|c|}
\hline \multirow{2}{*}{ Number of cases } & \multicolumn{2}{|c|}{ Present } & \multicolumn{2}{c|}{ Absent } & \multirow{2}{*}{ Total } \\
\cline { 2 - 5 } & $\mathbf{N}$ & $\mathbf{\%}$ & $\mathbf{n}$ & $\%$ & \\
\hline $1-2$ & 3 & 4.9 & 58 & 95.1 & 61 \\
\hline $3-6$ & 31 & 50.8 & 30 & 49.2 & 61 \\
\hline $7-15$ & 15 & 24.6 & 46 & 75.4 & 61 \\
\hline \&gt;15 & 12 & 19.7 & 49 & 80.3 & 61 \\
\hline \multicolumn{6}{|c|}{ Table-2: CECT N staging } \\
\hline
\end{tabular}

\begin{tabular}{|l|c|c|c|}
\hline \multirow{2}{*}{} & \multicolumn{2}{|c|}{ hi n 1-2nos } & \multirow{2}{*}{ Total } \\
\cline { 2 - 4 } & 1 & 0 & \\
\hline ct n 1-2no & 0 & 3 & 3 \\
0 & 7 & 51 & 58 \\
Total & 7 & 54 & 61 \\
\hline Fisher's Exact Test $p$ value $=0.386$; Sensitivity=0, Specifici- \\
ty=94.44 +ve predictive value=0, -ve predictive value=87.93 \\
\hline \multicolumn{2}{|c|}{ Table-3: Contingency table for CT vs histopathlogy findings of } \\
\multicolumn{2}{|c|}{ N1 disease (1= present, 0= absent) } \\
\hline
\end{tabular}

\begin{tabular}{|l|c|c|c|}
\hline \multirow{2}{*}{} & \multicolumn{2}{|c|}{ hi n 3-6nos } & \multirow{2}{*}{ Total } \\
\cline { 2 - 3 } & $\mathbf{1}$ & $\mathbf{0}$ & \\
\hline ct $\mathrm{n}$ 3-6no & 15 & 16 & 31 \\
0 & 12 & 18 & 30 \\
Total & 27 & 34 & 61 \\
\hline Pearson Chi-Square p value $=0.510$ Sensitivity=55.55 Specificity=52.94 \\
+ve predictive value=48.38, -ve predictive value=60 \\
\hline \multicolumn{2}{|c|}{ Table-4: Contingency table for CT vs histopathlogy findings of N2 } \\
disease (1= present, 0= absent) \\
\hline \multicolumn{2}{|c|}{} \\
\hline
\end{tabular}

\begin{tabular}{|l|c|c|c|}
\hline \multirow{2}{*}{} & \multicolumn{2}{|c|}{ hi $\mathbf{n ~ 7 - 1 5 n o s ~}$} & \multirow{2}{*}{ Total } \\
\cline { 2 - 3 } & $\mathbf{1}$ & $\mathbf{0}$ & \\
\hline ct $\mathrm{n}$ 7-15nos & & & \\
1.0 & 4 & 11 & 15 \\
0 & 18 & 28 & 46 \\
Total & 22 & 39 & 61 \\
\hline
\end{tabular}

Pearson Chi-Square $p$ value $=0.383$ Sensitivity $=18.18$ Specificity $=71.78+$ ve predictive value $=26.66$-ve predictive value $=60.86$

Table-5: Contingency table for CT vs histopathlogy findings of N3 disease ( $1=$ present, $0=$ absent $)$ 


\begin{tabular}{|l|c|c|c|}
\hline & \multicolumn{2}{|c|}{ hi $\mathbf{n}$ \&gt;15nos } & \multirow{2}{*}{ Total } \\
\cline { 2 - 3 } & $\mathbf{1}$ & $\mathbf{0}$ & \\
\hline Ct $\boldsymbol{n}$ \& $>$ 15nos & & & \\
1.0 & 3 & 9 & 12 \\
0 & 2 & 47 & 49 \\
Total & 5 & 56 & 61 \\
\hline
\end{tabular}

Fisher's Exact Test $p$ value $=0.036$ Sensitivity $=60$, Specificity=83.93, +ve predictive value $=25$,-ve predictive value=95.91

Table-6: Contingency table for CT vs histopathlogy findings of N3b disease ( $1=$ present, $0=$ absent $)$

(Table 3). CECT correctly identified involvement of N2 nodes in $15 / 27$ cases, giving a sensitivity of $55.55 \%$ and a specificity of $52.94 \%$. Operative assessment of N2 nodal involvement was similar to that of CT, correctly identifying $17 / 27$ cases (sensitivity $62.96 \%$ ), and a specificity of $23 / 34$ or $67.5 \%$ (Table 4).

Of the total 22 patients, CECT could identify only 4 with N3 nodal disease giving a sensitivity of 18.18 , but correctly excluded 28/39 patients without N3 disease (specificity 71.78\%) (Table 5 and 6).A total of 8 patients had liver involvement, confirmed by histopathological examination of the biopsies. CECT correctly detected 5 of them (sensitivity $62.5 \%$ ), but on 8 occasions CT provided a false positive report (specificity $84.91 \%$ ), which turned out to be inflammatory changes in 7 patients and 1 patient with a granulomatous lesion. CECT showed a sensitivity of $60 \%$ detecting only $3 / 5$ mesenteric deposits, and an equally low specificity of $78.57 \%$ excluding as many as 44 patients without disease. Fifty percent of patients with splenic invasion were detected by CECT (sensitivity 50\%) and excluded 53/57 patients without slpenic invasion (specificity $92.97 \%$ ). Of the total 11 patients who had omental involvement on histological examination, CECT detected only 3 cases (sensitivity 27.27), but could exclude 44/50 patients without omental disease (specificity 88\%). Surgical findings were superior than CT in detecting $7 / 11$ patients with omental deposits (sensitivity 63.63) and excluding all 50 patients without omental disease (specificity 100\%). Similar to detection of pelvic deposits, intra operative assessment for peritoneal deposits had 100\% sensitivity and specificity, where as CECT had only $33.33 \%$ sensitivity but with a comparable specificity of $96.15 \%$.

\section{DISCUSSION}

CECT is an inaccurate method of identifying lymph node metastases from gastric carcinoma. Our findings are consistent with those of other authors who have reported sensitivities ranging from 0 to $61 \% .10-12$ Detection of involved N1 lymph nodes was particularly poor, with a sensitivity of $0 \%$. CECT was reasonably specific, however, in the detection of uninvolved N1 nodes (specificity of 94.44\%). When perigastric nodes were examined, the results were consistent with that of Andaker et al. with a sensitivity of $26 \%$ and specificity of $99 \% .13$ The reason that sensitivity is so low and specificity so high is probably in part due to large cut-off size $(10 \mathrm{~mm})$ used to define "involved" nodes.

In other series, a smaller size was used than the $10 \mathrm{~mm}$ used in our series and any lowering of the size criterion will obviously permit identification of a greater proportion of malignant nodes, thus increasing sensitivity but at a cost of lowered specificity. The ability of spiral CT to identify involvement of $\mathrm{N} 2$ lymph nodes was better than for $\mathrm{N} 1$ nodes, the sensitivity being $55.55 \%$ and specificity of $52.94 \%$. Sendler et al have reported that CT is better at demonstrating involved N2 lymph nodes than involved N1 nodes for a number of reasons.14 Differentiation between involved nodes near the tumour and the primary tumour itself can be difficult. Nodes of normal size may contain metastatic deposits and enlarged N2 lymph nodes are more clearly distinguished from adjacent, highly enhancing vessels using the early phase of dynamic scanning. ${ }^{15}$

Preoperative knowledge of adjacent organ invasion and distant metastases is important in planning the operative procedure, allowing the surgeon to decide whether surgery is likely to be potentially curative or palliative in nature and also whether additional organs may have to be resected. Compared with previous reports of conventional CT, CECT scanning seems to be less sensitive but a more specific method for demonstrating invasion of both the small bowel and mesentry. CECT correctly identified only 2 of the 12 cases (sensitivity of $16.66 \%$ ) with invasion of the bowel and 3 of 5 cases (sensitivity 60\%) with mesentry involvement. The findings were in agreement with, Andaker et al13, who found conventional CT was insensitive in evaluating the invasion to colon and mesocolon, demonstrating this in only three of 12 cases with histological evidence of invasion (sensitivity $25 \%)$. Operative assessment was superior to spiral CT, with a sensitivity of $94 \%$ and specificity of $95 \%$.

The pancreas is an organ that has been notoriously difficult to assess both at laparotomy and on preoperative imaging. Previous reports have described sensitivities for assessment of pancreatic invasion varying from 27 to $95 \% .11$ In our hands, CECT scored a superior result at demonstrating invasion of the pancreas. Of the 7 patients with pancreatic invasion, 5 were correctly identified on CECT, whereas surgical assessment correctly identified all 7 patients. Both CECT and surgical assessment were highly specific, but operative assessment was better. CT visualization of the pancreas can be difficult in patients with gastric cancer as they are frequently malnourished and visceral fat between the organs is depleted.14 Although a clear fat plane between stomach and pancreas can reliably be taken to indicate noninvasion, the absence of a fat plane between these organs does not necessarily imply invasion. In similar fashion, irregularity of the interface does not necessarily represent invasion.15 Differentiation between inflammatory adhesion, oedema and true invasion, both on CECT and at operation, can be extremely difficult. 11 In this study, both false positive operative assessments were found to represent inflammatory reaction on subsequent histological examination. CECT has the potential to demonstrate both liver and peritoneal metastases because it can be used to examine the entire abdominal cavity, unlike EUS.16 CECT correctly demonstrated 5/8 cases with liver metastases (sensitivity 62.5\%). These metastases were identified at operation and confirmed histologically. It is important to remember that for assessment of hepatic metastases, the CT findings were compared against the operative findings confirmed histologically. However, there 
may have been other small intrahepatic metastases which were not identified at operative assessment. This indicates that there could have been a number of false negative cases that were not identified. Ideally intra-operative ultrasound has been used as a gold standard for detecting hepatic metastases. ${ }^{16}$

It has been reported previously that CT is limited in the demonstration of peritoneal metastases, being able to identify peritoneal disease only in the presence of ascites. ${ }^{16}$ Whilst CT can identify obvious gross peritoneal disease, with large omental cake-like deposits, it is poor at visualizing small individual peritoneal nodules due to its limits of resolution. In our study, CECT correctly demonstrated 3 of the 9 cases only (sensitivity $33.33 \%$ ) in which peritoneal metastases were found at sites separate from the main tumour. It comes as no surprise that $\mathrm{CT}$ is poor at predicting the $\mathrm{T}$ stage of disease in patients with gastric cancer. Although $\mathrm{CT}$ was able to demonstrate the stomach wall as a three layer structure corresponding to the mucosal, submucosal and musculoserosal layers, the outer musculo-serosal layer (thin in places) cannot always be visualized. ${ }^{16}$ Destruction of this layered structure together with gastric wall thickening may be seen on scanning, although correct assignment to a T stage (T1T3) may be extremely difficult or even impossible. In our study, all of our patients presented late with lesions already in T4 stage. This, together with limited ability of CT to identify lymph node metastases, means that this modality is of limited value as a predictor of overall stage of gastric cancer. From this study, however, it is clear that due to high specificity, when CT demonstrates an "uninvolved" node, it is very likely not to contain tumour on histological examination. Of most concern from recent reports is the suggestion that CT can over-stage patients, by falsely predicting the presence of irresectable disease which was not confirmed at subsequent laparotomy. In this series, CECT was found to be highly specific, which meant that there was a low incidence of false positive findings.

For surgeons who practise radical "D2" resections, the most important information required is whether distant metastatic (M1) disease is present, which implies that a potentially curative resection cannot be performed.

\section{CONCLUSION}

CECT remains poor at identifying lymph node metastases and in determining depth of tumour invasion (T1-T3), but was highly specific which meant that a positive spiral CT result could be relied upon. It was also of value in identifying invasion of adjacent organs and some cases with distant metastatic (M1) disease. At present, we perform CECT routinely on all patients and adopt a selective staging laparoscopy/ mini laprotomy policy in those patients in whom the status of the peritoneal cavity and liver remains in doubt.

\section{REFERENCES}

1. Siegel R, Ma J, Zou Z, Jemal A. Cancer statistics, 2014. CA Cancer J Clin. 2014;64(1):9-29.

2. Hirayama T. Epidemiology of cancer of the stomach with special reference to its recent decrease in Japan.
Cancer Res. 1975;35(5):3460-3.

3. Map [Internet]. Available from:http://globocan.iarc.fr/ Pages/Map.aspx\#

4. Maconi G, Kurihara H, Panizzo V, Russo A, Cristaldi M, Marrelli D, et al. Gastric cancer in young patients with no alarm symptoms: focus on delay in diagnosis, stage of neoplasm and survival. Scand J Gastroenterol. 2003; 38(3):1249-55

5. Stephens MR, Lewis WG, White S, Blackshaw GRJC, Edwards P, Barry JD, et al. Prognostic significance of alarm symptoms in patients with gastric cancer. $\mathrm{Br} \mathrm{J}$ Surg. 2005;92(6):840-6.

6. Bowrey DJ, Griffin SM, Wayman J, Karat D, Hayes $\mathrm{N}$, Raimes SA. Use of alarm symptoms to select dyspeptics for endoscopy causes patients with curable esophagogastric cancer to be overlooked. Surg Endosc. 2006;20(2):1725-8.

7. Fein R, Kelsen DP, Geller N, Bains M, McCormack $\mathrm{P}$, Brennan MF. Adenocarcinoma of the esophagus and gastroesophageal junction. Prognostic factors and results of therapy. Cancer. 1985;56(4):2512-8.

8. Rey-Ferro M, Castaño R, Orozco O, Serna A, Moreno A. Nutritional and immunologic evaluation of patients with gastric cancer before and after surgery. Nutrition.1997;13(1):878-81.

9. Coggon D, Barker DJ, Cole RB, Nelson M. Stomach cancer and food storage. J Natl Cancer Inst. 1989;81(1):1178-82.

10. Dehn TC, Reznek RH, Nockler IB, White FE. The pre-operative assessment of advanced gastric cancer by computed tomography. Br J Surg. 1984;7(4):413-7.

11. Ziegler K, Sanft C, Zimmer T, Zeitz M, Felsenberg D, Stein H, Germer C, Deutschmann C, Riecken EO.Comparison of computed tomography, endosonography and intraoperative assessment in TN staging of gastric carcinoma. Gut. 1993;34(5):604-10.

12. Andåker L, Morales $\mathrm{O}$, Höjer H, Bäckstrand B, Borch K, Larsson J. Evaluation of preoperative computed tomography in gastric malignancy. Surgery. 1991;109(2):132-5.

13. Kleinhaus U, Militianu D. Computed tomography in the preoperative evaluation of gastric carcinoma. Gastrointest Radiol. 1988;13(4):97-101.

14. Tsuburaya A, Noguchi Y, Matsumoto A, Kobayashi S, Masukawa K, Horiguchi K. A preoperative assessment of adjacent organ invasion by stomachcarcinoma with high resolution computed tomography. Surg Today. 1994;24(3):299-304.

15. Sendler A, Dittler HJ, Feussner H, Nekarda H, Bollschweiler E, Fink U, Helmberger H, Höfler H, Siewert JR. Preoperative staging of gastric cancer as precondition for multimodaltreatment. World J Surg. 1995;19(1):501-8.

16. Minami M, Kawauchi N, Itai Y, Niki T, Sasaki Y. Gastric tumors: radiologic- pathologic correlation and accuracy of $\mathrm{T}$ stagingwith dynamic CT. Radiology. 1992;185(2):173-8.

\section{Source of Support: Nil; Conflict of Interest: None}

Submitted: 24-08-2019; Accepted: 15-10-2019; Published online: 13-11-2019 